\title{
Kranion EDITORIAL
}

\section{Prevención del deterioro cognitivo leve y la demencia: estamos a tiempo Prevention of mild cognitive impairment and dementia: We still have time}

\author{
David Ezpeleta \\ Servicio de Neurología, \\ Hospital Universitario Quirónsalud Madrid, Madrid
}

«Si pudiésemos dar a cada individuo la cantidad adecuada de nutrición y ejercicio, ni muy poco ni demasiado, habríamos encontrado el camino más seguro hacia la salud». Hipócrates de Cos (460 a.C. - 370 a.C.)

La demencia es un problema de salud pública creciente que afecta a alrededor de 50 millones de personas en todo el mundo. Hay casi 10 millones de casos nuevos cada año y se prevé que esta cifra se triplique en $2050^{1}$. Actualmente, unas 800.000 personas padecen enfermedad de Alzheimer (EA) en España, cada año se diagnostican unos 40.000 nuevos casos y su coste supone el 1,5\% del producto interior bruto nacional ${ }^{2}$. El peso que tienen 12 factores de riesgo modificables supone un 40\% de los casos. En una situación ideal, podríamos retrasar o prevenir el $40 \%$ de las demencias interviniendo precozmente en dichos factores ${ }^{3}$.

Las proyecciones de incidencia y prevalencia de EA y otras demencias auguran un medio plazo que supondrá un reto a todos los niveles. Al paulatino aumento de personas con demencia se añade el deterioro cognitivo ligero (DCL), motivo de consulta de frecuencia creciente en el ámbito especializado. La posibilidad de hacer un diagnóstico precoz y específico mediante biomarcadores de neuroimagen molecular, líquido cefalorraquídeo, sangre periférica e incluso biomarcadores digitales abre la puerta al tratamiento precoz. Sin embargo, mientras no se disponga de tratamientos realmente eficaces y sostenibles en el tiempo por los sistemas de salud tendremos un problema añadido de doble vertiente. Por un lado, habrá que dotar a los neurólogos subespecializados en conducta, DCL, EA y otras demencias de los espacios físicos, medios diagnósticos y terapéuticos, así como de los recursos humanos necesarios, algo que hoy por hoy solo se aguanta sobre el papel. Por otro lado, surgen numerosas cuestiones de índole ética, legal y práctica. Los horizontes 2023 y 2025 de los Planes de Acción frente a la EA y otras demencias del Ministerio de Sanidad, Consumo y Bienestar Social del Gobierno de España y la Organización Mundial de la Salud (OMS), respectivamente, son un buen comienzo, pero los plazos deben ampliarse y, sobre todo, las intenciones y compromisos que ambos documentos recogen hay que ponerlos en práctica sin dilación ${ }^{4,5}$.

Siendo cierto que el diagnóstico precoz y sobre todo específico es algo actualmente posible en fases prodrómicas e incluso preclínicas, un hito perseguido durante décadas y deseado por todos, deberíamos ofrecer una contrapartida terapéutica. Formar parte de una cohorte, entrar en un ensayo clínico o hacer recomendaciones relacionadas con el estilo de vida y los factores de riesgo vascular no parece ser suficiente. Los problemas ético-legales del diagnóstico

\section{Autor de correspondencia:}

David Ezpeleta

E-mail: neuroezpeleta@gmail.com
Fecha de recepción: 20-11-2021

Fecha de aceptación: 29-11-2021

DOI: 10.24875/KRANION.M21000011
Disponible en internet: 24-12-2021

Kranion. 2021;16:77-8

www.kranion.es

1577-8843 / (c) 2021 Kranion. Publicado por Permanyer. Este es un artículo open access bajo la licencia CC BY-NC-ND (http://creativecommons.org/licenses/by-nc-nd/4.0/). 
predictivo preclínico y prodrómico fueron analizados en detalle por Gil-Navarro en un reciente número de esta revista ${ }^{6}$. A juicio de esta autora, que compartimos, la posible estigmatización, aislamiento, despido laboral, abuso económico 0 discriminación en el acceso a recursos adquiere matices diferentes para la persona en riesgo, su familia y los profesionales sanitarios, legisladores y poderes públicos. El diagnóstico predictivo debe dotarse de un marco ético-legal que permita un adecuado seguimiento de la pérdida de la capacidad cognitiva en la realización de las actividades cotidianas, preservando la autonomía de las personas y su derecho a la confidencialidad ante las consecuencias de una EA todavía en sus albores ${ }^{6}$.

Así las cosas, a la espera de terapias que modifiquen de forma franca y clínicamente significativa el curso natural de estas enfermedades, y mientras se dimensiona y dota de todos los recursos necesarios a los sistemas de salud en tiempo y forma, la prevención entra en escena y cobra protagonismo. No se trata de la opción de la derrota, el consuelo y comodín del mientras tanto, el algo hay que hacer. Es, seguramente, la clave para que tales objetivos sean posibles y sostenibles en el tiempo.

Las directrices de la OMS para la reducción del riesgo de deterioro cognitivo y demencia, publicadas en 2019, establecen las siguientes intervenciones y estrategias terapéuticas, cada una con su calidad de la evidencia y fuerza de la recomendación: actividad física regular, abandono del consumo de tabaco, intervenciones nutricionales, intervenciones relacionadas con el consumo nocivo de alcohol, intervenciones relacionadas con la capacidad cognitiva, actividad social, control de la obesidad y tratamiento de la hipertensión, diabetes, dislipidemia, depresión y pérdida de audición ${ }^{1}$. Hipócrates, hace 24 siglos, adelantó los factores de riesgo más importantes por sus contrarios: "la cantidad adecuada de nutrición y ejercicio, ni muy poco ni demasiado». Si bien se ha estimado el peso de cada factor de riesgo modificable en el desarrollo de demencia, los estudios de intervenciones aisladas, salvo el ejercicio ${ }^{7-9}$, no han reflejado un beneficio equiparable. Por ello, la estrategia actual es la prevención multimodal, tanto en el ámbito de los ensayos clínicos como en su puesta en práctica mientras se esperan resultados, que pueden tardar en llegar debido a la complejidad de este tipo de estudios. Todos estos aspectos se tratan con detalle en las sobresalientes y generosas revisiones de este número de la revista que, al alimón, publican las doctoras Manzano y Terrón.

La prevención del DCL y las demencias lo es también de las enfermedades vasculares y del cáncer, pues comparten muchos factores de riesgo. Nunca es tarde para mejorar el estado de salud de las personas, pero el objetivo primero y último de toda estrategia preventiva es evitar que las cosas sucedan. Sin embargo, las intervenciones preventivas, a muy largo plazo y de ámbito poblacional, son mucho más complejas de llevar a cabo con éxito que el tratamiento de la enfermedad diana. Los humanos somos animales de costumbres y, pasados unos años, la resistencia a los cambios de hábitos es creciente. Hay que comenzar durante la infancia y la adolescencia, no queda otra.

Hace unos meses tuvimos la inmensa fortuna de colaborar en un proyecto docente extraordinario, disruptivo y singular: un musical creado, guionizado y ejecutado por los alumnos de educación secundaria obligatoria (ESO) de una ikastola de Pamplona. El protagonista es un payaso que sufre Alzheimer. La propuesta, avalada por la Sociedad Española de Neurología ${ }^{10}$, se representó en grandes salas de conciertos, centros educativos y centros de mayores. Además, se prepararon unidades didácticas sobre conocer, identificar, prevenir y actuar en relación con la EA y, por extensión, otras demencias, en todos los casos adaptadas a los diferentes niveles educativos de infantil, primaria y ESO. Dedicaremos un trabajo a explicar en detalle este milagro en un próximo número de la revista.

Toda manipulación social (en nuestro caso, intervención poblacional virtuosa) que se precie necesita como mínimo de una generación. La prevención de las principales enfermedades en general, y de las demencias en particular, justifica per se la implantación de una asignatura escolar obligatoria. No se nos ocurre mejor forma para, aun llegando tarde, llegar a tiempo.

\section{BIBLIOGRAFÍA}

1. World Health Organization (WH0). Risk reduction of cognitive decline and dementia. WHO Guidelines [Internet]. World Health Organization; 2019 [consultado: 25 de noviembre de 2021$].$ Disponible en: https://apps.who.int/iris/bitstream/handle/10665/312180/9789241550543-eng.pdf

2. 21 de septiembre: Día Mundial de la Enfermedad de Alzheimer. El 35\% de los casos de Alzheimer se pueden atribuir a nueve factores de riesgo modificables [Internet]. Sociedad Española de Neurología [consultado: 25 de noviembre de 2021]. Disponible en: https://www.sen.es/saladeprensa/pdf/Link280.pdf

3. Livingston G, Huntley J, Sommerlad A, Ames D, Ballard C, Banerjee S, et al. Dementia prevention, intervention, and care: 2020 report of the Lancet Commission. Lancet. 2020; 396(10248):413-46

4. Plan Integral de Alzheimer y otras Demencias (2019-2023) [Internet]. Gobierno de España, Ministerio de Sanidad, Consumo y Bienestar Social; 2019 [consultado: 26 de noviembre de 2021]. Disponible en: https://www.mscbs.gob.es/profesionales/saludPublica/docs/Plan_Integral_Alhzeimer_Octubre_2019.pdf

5. Global action plan on the public health response to dementia 2017-2025 [Internet]. World Health Organization; 2017 [consultado: 26 de noviembre de 2021$]$. Disponible en: https:// www.who.int/publications/i/item/9789241513487

6. Gil-Navarro S. Indicaciones y consideraciones eticolegales en el uso de biomarcadores en demencias neurodegenerativas. Kranion. 2020;17:129-35.

7. Müller S, Preische 0, Sohrabi HR, Gräber S, Jucker M, Ringman JM, et al. Relationship between physical activity, cognition, and Alzheimer pathology in autosomal dominant Alzheimer's disease. Alzheimer's Dement. 2018;14(11):1427-37.

8. Hörder H, Johansson L, Guo X, Grimby G, Kern S, Östling S, et al. Midlife cardiovascular fitness and dementia. Neurology. 2018;90(15):e1298-305.

9. González García MN. Ejercicio, cerebro y enfermedades neurológicas. Kranion. 2019;14:136-41.

10. Musical Ximur, dedicado a la enfermedad de Alzheimer [Internet]. Sociedad Española de Neurología; 2021 [consultado: 26 de noviembre de 2021]. Disponible en: https://www.sen.es/ noticias-y-actividades/otras-convocatorias-y-actividades/2889-musical-ximur-dedicado-a-la-enfermedad-de-alzheimer. Con acceso el 26 de noviembre de 2021. 